\title{
Influence of the relative rotational speed on component features in micro rotary swaging
}

\author{
Svetlana Ishkina ${ }^{a}$, Bernd Kuhfuss, and Christian Schenck \\ Bime Bremen Institut for Mechanical Engineering, University of Bremen, Bremen, Badgasteiner \\ Strasse 1, 28359, Germany
}

\begin{abstract}
Micro rotary swaging is a cold forming process for production of micro components with determined geometry and surface. It is also possible to change the microstructure of wires and hence the material properties. Swaging dies revolve around the work piece with an overlaid radial oscillation. Newly developed tools (Flat Surface Dies, FSD) feature plain surfaces and do not represent the geometry of the formed part as in conventional swaging. Using these tools allows for producing wires with triangle geometry (cross section) as well as a circular shape. To test the influence of FSD on material properties by micro swaging a new method is investigated: the variation of the relative speed between the specimen and dies in infeed rotary swaging. During this specific process copper (C11000) and steel (304 Alloy) wires with diameter $\mathrm{d}_{0}=1 \mathrm{~mm}$ are formed. It is noticed that the mechanical characteristics such as ductility and strength differ from the characteristics after conventional swaging. Moreover this approach enables new possibilities to influence the geometry and the surface quality of wires. The impact of the relative speed on the processed wire features is described in this paper.
\end{abstract}

\section{Introduction}

Rotary swaging is an incremental cold forming process especially for wires. The geometry is achieved with consecutive small forming steps. The shape (cross section) of the workpieces after micro rotary swaging has fundamentally a round geometry with diameters less than $1 \mathrm{~mm}$, that corresponds to the CIRP-definition of micro parts [1]. Micro rotary swaging process changes also the material properties of wires. Moumi found that in cold worked wires of 304 alloy the strain decreases and the ultimate tensile strength increases more than twice compared to the initial values [2]. He also described how a variation of the swaging parameters such as the feed velocity and the number of forming increments influences properties and microstructure of copper wires [3]. Bruder introduced in [4] a new Equal Channel Angular Swaging (ECAS) process in macro range. ECAS allows continuous production of bulk ultra fine grain (UFG) material with significant refinement of the microstructure due to shear deformation during swaging. Grain size modification of wires was also shown in [5]. An influence of new developed swaging tools and specific process with asymmetrical strokes of the forming dies on

\footnotetext{
${ }^{\text {a }}$ Corresponding author: ishkina@bime.de
}

This is an Open Access article distributed under the terms of the Creative Commons Attribution License 4.0, which permits unrestricted use, distribution, and reproduction in any medium, provided the original work is properly cited. 


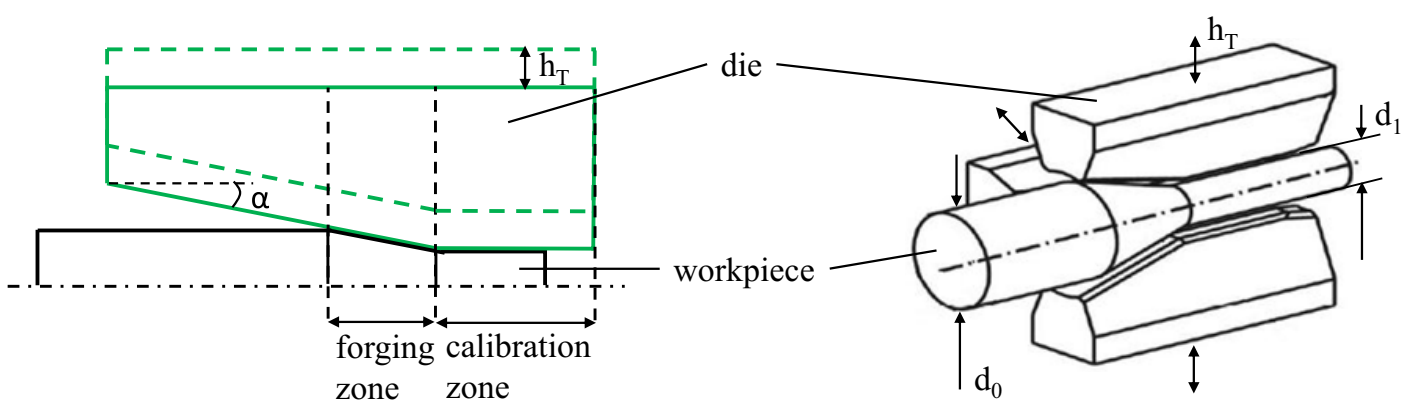

Figure 1. Schematic of the infeed rotary swaging.

the material properties was studied. It was found that unconventional settings of the swaging head in combination with Flat Swaging Dies (FSD) increase the shear strain in the wire and affect mechanical properties, grain size evolution and the geometry of wires.

In order to test an influence of FSD on processed wire features by swaging a special rotary swaging method based on infeed rotary swaging is investigated: the variation of the relative speed between the specimen and dies. In the infeed rotary swaging process (see Fig. 1) the workpiece is fed axially into the swaging head and the reduction of the workpiece starts in the forging zone of the forming dies, with further forming in the calibration zone. The tools revolve around the work piece and oscillate radially with a small amplitude $\mathrm{h}_{\mathrm{T}}$ [3]. In new Relative Speed Swaging (RSS) process an angular velocity of the tools $\omega_{1}$ was constant for each experiment and the workpiece rotated in the same direction with a definite angular velocity $\omega_{2}$. The relative speed $\Delta \omega$ is defined as the difference between the angular velocity of the tools $\Delta \omega_{1}$ and the angular velocity of the workpiece $\Delta \omega_{2}\left(\Delta \omega=\omega_{1}-\omega_{2}\right)$.

The purpose of this paper is to examine how the combination of the FSD with RSS affects mechanical properties, grain size development and the geometry of wires. The first part explains the setup of experiments. The second part includes experimental results and discussions.

\section{Experimental setup}

During the rotary swaging process copper and stainless steel wires with an initial diameter of $\mathrm{d}_{0}=1 \mathrm{~mm}$ were formed. The workpieces were fed continuously to the swaging head by a linear direct drive with a feed velocity $\mathrm{v}_{\mathrm{f}}=1 \mathrm{~mm} / \mathrm{s}$ (copper) and $\mathrm{v}_{\mathrm{f}}=0.5 \mathrm{~mm} / \mathrm{s}$ (steel).

Swaging tools consist of three swaging dies. The die angle $\alpha$ of the Curved Swaging Dies (CSD) measures $10^{\circ}$ and the calibration zone is $20 \mathrm{~mm}$ long. The newly developed tools feature flat surfaces in the calibration zone instead of the conventional curved ones. However, the reduction zone of the Flat Swaging Dies (FSD) provides a light curved surface which turns into a flat surface of the calibration zone. The radius of the FSD reduction zone is larger than that of CSD. The die angle $\alpha$ of the FSD is equal to $5^{\circ}$ and the calibration zone measures $8 \mathrm{~mm}$. The strokes of all swaging dies were equal to $\mathrm{h}_{\mathrm{T}}=0.4 \mathrm{~mm}$ for each experiment. Furthermore, CSD and FSD do not provide equal height $\mathrm{H}_{\mathrm{d}}$ (see Fig. 2(a) and (b)). The levelling of $\mathrm{H}_{\mathrm{d}}$ was difficult in the presented tests and the closing pressure is expected to be different with the alternative tools.

The variation of the relative speed between the specimen and dies in infeed rotary swaging was realised by different rotation speeds of the workpiece. Angular velocity of the tools was kept constant at $\omega_{1}=89.23 \mathrm{rad} / \mathrm{s}$, what results in a stroke frequency of $\mathrm{f}_{\mathrm{st}}=102 \mathrm{~Hz}$.

Experiment I was carried out by CSD with $\omega_{2}=0$ (see Fig. 3(a)). All experiments with FSD and RSS had the same settings but different angular velocities. The difference of the angular velocity in Experiment II was equal to $\Delta \omega=0 \mathrm{rad} / \mathrm{s}$, in Experiment III it was equal to $\Delta \omega=66.93 \mathrm{rad} / \mathrm{s}$. It means 


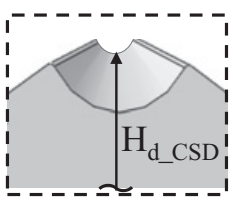

(a)

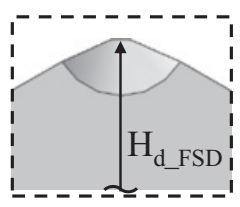

(b)

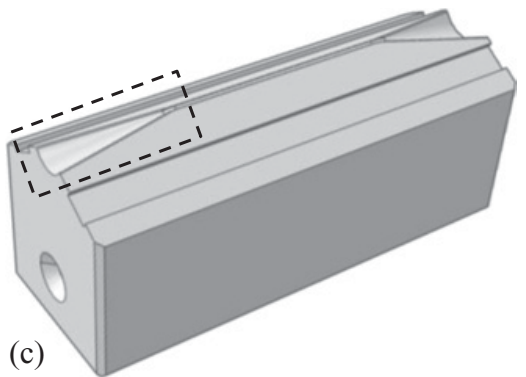

Figure 2. Swaging die (a) curved (CSD) and (b) flat (FSD); (c) overview.

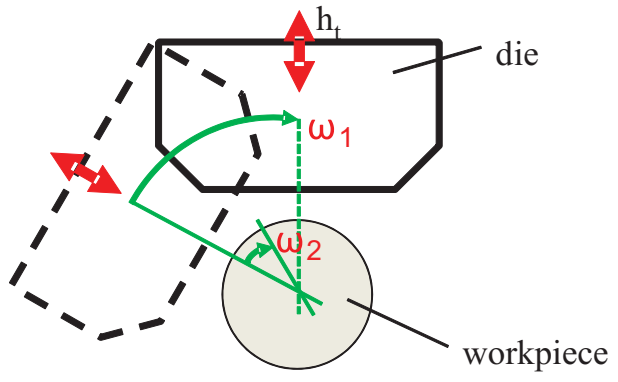

(a)

\begin{tabular}{|c|c|c|}
\hline Experiment & Die & $\Delta \boldsymbol{\omega}[\mathrm{rad} / \mathrm{s}]$ \\
\hline I & CSD & 89.23 \\
\hline II & FSD & 89.23 \\
\hline III & FSD & 66.93 \\
\hline IV & FSD & 44.62 \\
\hline
\end{tabular}

(b)

Figure 3. Experiments a) scheme, b) parameters.

that the workpiece rotates with the angular velocity of $\omega_{2}=22.30 \mathrm{rad} / \mathrm{s}$, that is four times slower as tools rotation. Experiment IV reflects the geometry of the wires after swaging with $\Delta \omega=44.62 \mathrm{rad} / \mathrm{s}$ while the angular velocity of the workpiece was $\omega_{2}=44.61 \mathrm{rad} / \mathrm{s}$, that is two times slower as the velocity of the swaging tools. Such differences are intended to increase the shear component of plastic strain. The parameters of the experiments are summarised in Fig. 3(b).

\section{Results and discussion}

\subsection{Experimental results}

During the process the copper and stainless steel wires with an initial diameter of $\mathrm{d}_{0}=1 \mathrm{~mm}$ were formed. The geometry (cross section) of the wires after conventional swaging with CSD (I) and after new method using FSD (II, III and IV) is shown in Fig. 4 for copper wires and Fig. 5 for steel. The cross section of II shows that the wires formed with FSD have a sufficient roundness. The diameter I and II are not equal what can be explained by the changed closing pressure. Figure 4 (II-V) shows that the diameter and the shape of wires are changed with the variation of $\Delta \omega$.

Experimental rotation tests of the workpiece and tools revealed a standard deviations of the relative speed $\Delta \omega$ for copper experiments of maximum $\sigma_{\Delta \omega_{-} \max }=0.0105 \mathrm{rad} / \mathrm{s}$ and for steel $\sigma_{\Delta \omega_{-} \max }=$ $0.0110 \mathrm{rad} / \mathrm{s}$. This scatter is acceptable low.

The effect of extraordinary geometry for III and IV is not so obvious for the formed steel wires. The geometry and the diameter of the cross sections do not differ significantly (see Fig. 5 (II-IV)).

The influence of the the die angle $\alpha$ is not explicitly analyzed in this study. In order to examine the geometry of the wires quantitatively the profile and the roughness $\mathrm{R}_{\mathrm{a}}$ of the formed wires were measured 


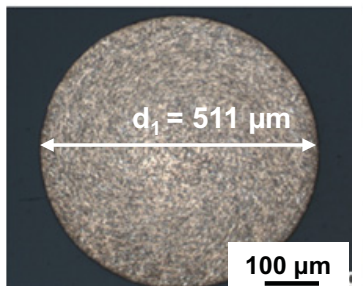

I

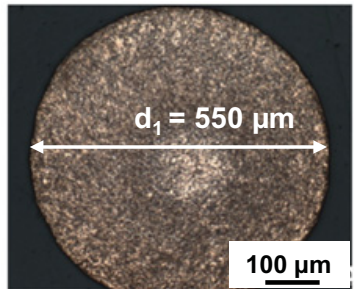

II

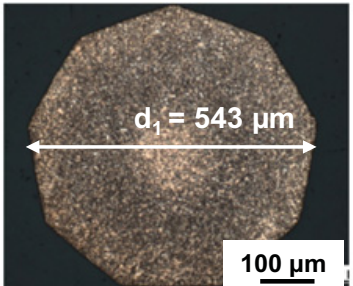

III

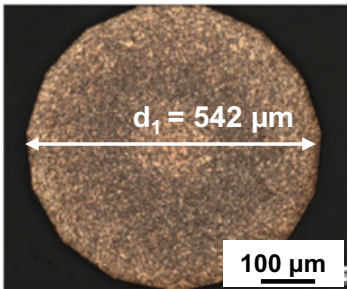

IV

Figure 4. Cross section of the copper wires.

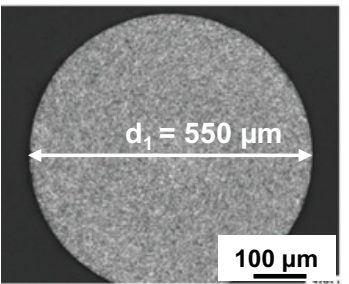

I

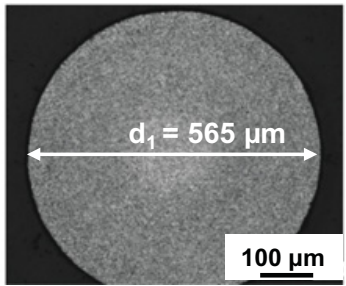

II

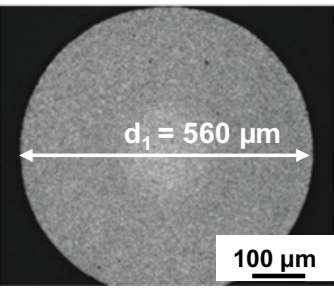

III

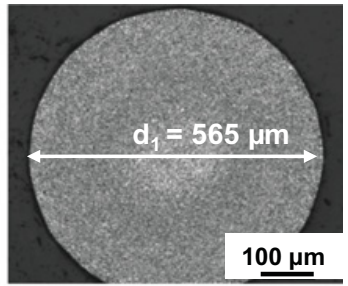

IV

Figure 5. Cross section of stainless steel wires.

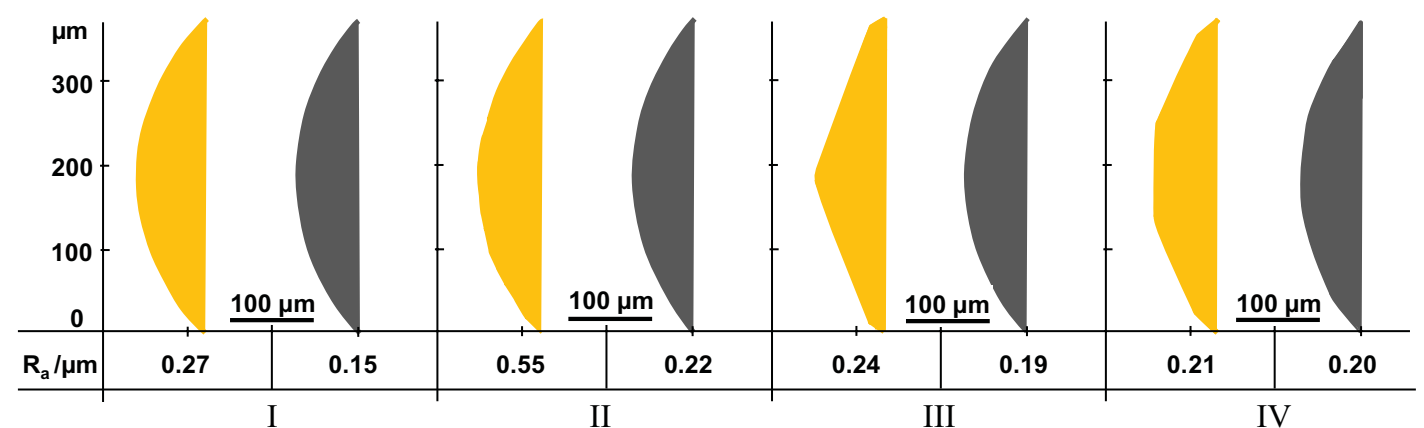

Figure 6. The profile and an arithmetic average of absolute values $\mathrm{R}_{\mathrm{a}}$ of the copper wires (left) and stainless steel wires (right).

with a high resolution optical microscope (see Fig. 6). The wires formed with CSD have a better surface then with FSD in experiment II which can be explained with the longer calibration zone of the forming dies.

\subsection{Mechanical characteristics}

Tensile tests were carried out with initial and formed wires. The circles and triangle marks in Fig. 7 show the experimental data for yield strength at $0.2 \%$ and the total elongation. After rotary swaging the yield strength increases 2-3 times while the total elongation decreases from about $45 \%$ for copper and $50 \%$ for steel to less than $2 \%$ for both materials. The total elongation of the formed copper wires 

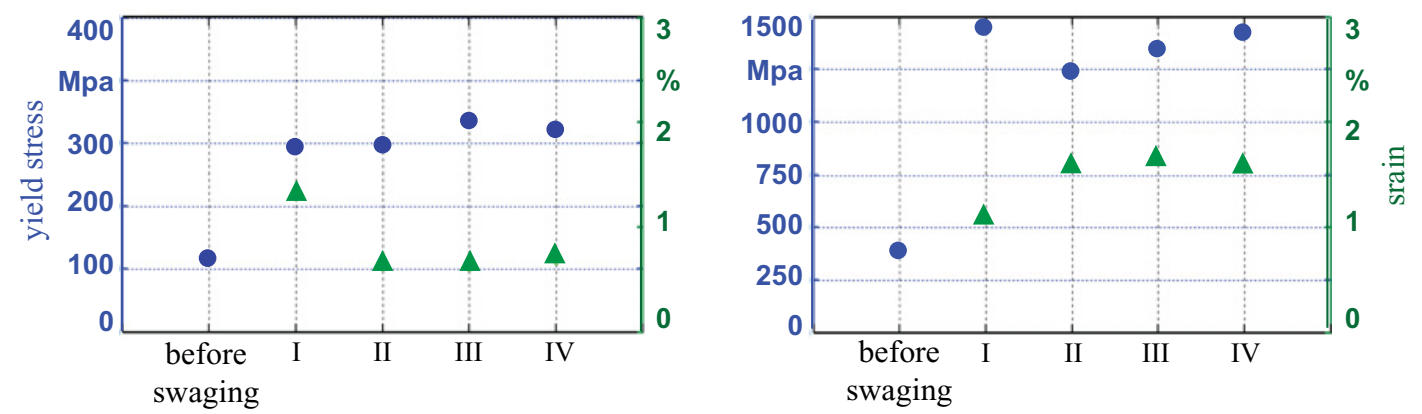

Figure 7. Yield stress and strain values of the initial and formed wires for copper (left) and stainless steel (right).

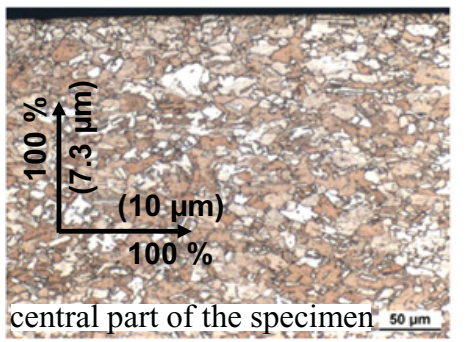

before swaging

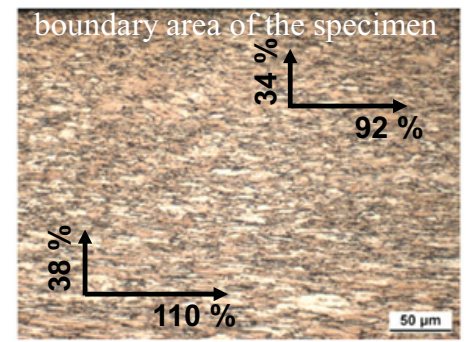

III

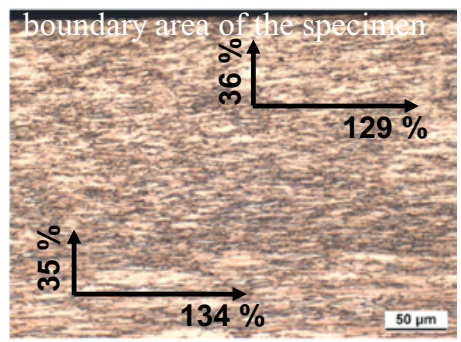

IV

Figure 8. Microstructure of copper wires (longitudinal section).

with CFD (see Fig. 7 (I) left) is higher than after swaging with FSD and different angular velocities (see Fig. 7 (I-IV) left).

Another effect can be observed in Fig. 7 (right) regarding to the formed steel wires. The value of total elongation after RSS process (II-IV) is higher than that after conventional swaging (I). The yield strain at $0.2 \%$ after conventional swaging for copper and steel wires differs insignificantly from the $(0.2 \%)$ yield stress of the wires formed by relative speed variation.

\subsection{Grain size evolution}

Microstructure of the initial and swaged copper specimens was also examined with an optical microscope. For the analysis of the average grain size, the ASTM-E112 test method was applied; the grain size was determined both in radial and axial direction.

In Fig. 8 the microstructure of the specimen before cold forming and after RSS process is shown. The average grain size of the copper specimen at the initial stage is $7.3 \mu \mathrm{m}$ in the radial direction and about $10.0 \mu \mathrm{m}$ in the axial direction. Swaging of copper wires with experiment III gives an opportunity to reduce the grain size in the radial direction more as twice at approximately unchanged grain size in the axial direction. The radial value of the grain size is reduced to $34 \%$ in the boundary area and to $38 \%$ in the central part of the specimen. The radial grain size of the microstructure in experiment IV is similar to that for III, while an elongation of the grains in axial direction is about $30 \%$ in both areas.

Figure 9 (left) shows the microstructure of stainless steel before forming with a mean value of grain sizes of $5.3 \mu \mathrm{m}$ in the radial direction and about $5.6 \mu \mathrm{m}$ in the axial direction. Unlike the formed copper wires an intense grain elongation after new method III for steel wires is observed. The grain size is 


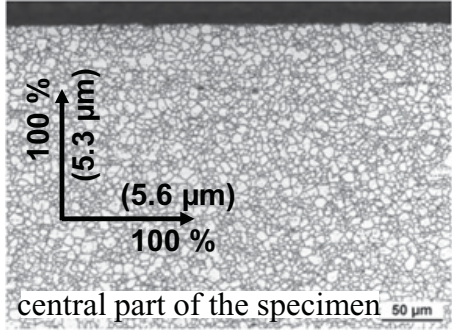

before swaging

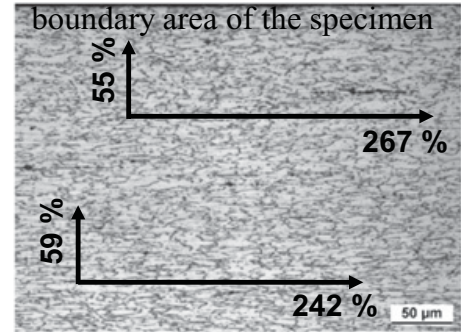

III

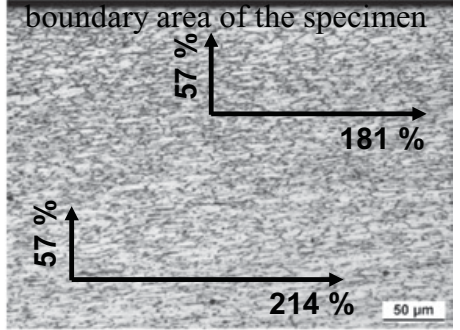

IV

Figure 9. Microstructure of stainless steel wires (longitudinal section).

almost twice thinner in the radial direction and has a value of $55 \%$ in the boundary area of the specimen and 59\% in the central part; thereby it is nearly more as twice longer in the axial direction. The results obtained for the wires formed with RSS process, FSD (experiment IV) are similar to the results of grain sizes showed in Fig. 9 (III) in the radial direction while the grains are $81 \%$ longer in the boundary area and $114 \%$ longer in the central part of the specimen.

The analysis of the microstructure of both materials showed that the modification of grain size in copper and stainless steel was achieved through the variation of the angular velocity of the workpiece and the use of FSD. The grain size of the cross section corresponds to the grain size in the radial direction of longitudinal section as in a central part of the specimen as in the boundary area.

\section{Conclusion and outlook}

A new process based on rotary swaging is introduced and analysed in this study. Conventional used dies reproduce their geometry in the workpiece. They are replaced by flat surface dies that enable the forming of different shapes and dimensions with one set of tools. In combination with a controlled relative rotation of tools and workpiece the material is churned and hence, the microstructure is influenced. In consequence besides the processed cross sections the material properties like yield stress and strain are adjustable by the new process parameter $\Delta \omega$. Experiments were conducted with copper and stainless steel wires. The yielded surface roughness was quite good with values between $R_{\text {a_best }}=0.15 \mu \mathrm{m}$ and $\mathrm{R}_{\mathrm{a} \_ \text {worst }}=0.55 \mu \mathrm{m}$. With copper a higher diversity of resulting cross section shapes is possible whereas the change of the material properties is higher with steel.

The initial goal of this study was to improve the formability of the wires for further cold forming processes by creating a ultra-fine grained microstructure which can be achieved by severe plastic deformation processes [6]. The presented proceeding and the results are encouraging that the rotary swaging can be evolved as an alternative process for producing UFG wires with high dimensional accuracy. Next steps in the research will be the analysis of higher deformation degrees and the combination of the relative speed approach with the idea of non-rotation-symmetric strokes [5].

The authors would like to thank the German Research Foundation (DFG) for financial support of the project T2 within the collaborative research centre SFB 747 "Micro Cold Forming".

\section{References}

[1] M. Geiger, M. Kleiner, R. Eckstein, N. Tiesler, U. Engel, Ann. CIRP 50, 445-462 (2014)

[2] E. Moumi, B. Kuhfuss, Proc. 4th Int. Conf. on Nanomanuf. (2014) 


\section{ICNFT 2015}

[3] E. Moumi, B. Kuhfuss, B. Koeler, H. Bomas, Proc. 8th Int. Conf. on Micromanuf. (2013)

[4] E. Bruder, M.O. Görtan, P. Groche and C. Müller, Mater.Sci. Forum 667-669, 103-107 (2011)

[5] S. Ishkina, B. Kuhfuss, C. Schenck, Proc. 18th Int. Conf. on Mat. Form. ESAFORM. Published in Key Eng. Mat. 651-653, 627-632 (2015)

[6] R.Z. Valiev, R.K. Islamgaliev, I.V. Aleksandrov, Prog. Mat. Sci. 45, 103-189 (2000) 